\title{
INTERNACIONALIZAÇÃO DO PSICODRAMA BRASILEIRO
}

\section{INTERNATIONALIZATION OF BRAZILIAN PSYCHODRAMA \\ INTERNACIONALIZACIÓN DEL PSICODRAMA BRASILEÑO \\ Heloisa Junqueira Fleury ${ }^{1, *}$}

A Revista Brasileira de Psicodrama está completando 30 anos em 2022, uma oportunidade para celebrar o seu fortalecimento com um periódico científico e para avaliar as possibilidades de novos avanços para a próxima década.

Muitos psicodramatistas reconhecidos pela excelência de suas práticas e do ensino do psicodrama evitam o desafio de escrever e publicar seus artigos, tornando o psicodrama brasileiro menos conhecido em outros continentes. Essa ausência de artigos brasileiros reflete, além da pouca familiaridade com a escrita científica, a dificuldade para a pesquisa e publicação em inglês.

A diversidade cultural e linguística é fundamental, especialmente em psiquiatria infantil e do adolescente, em que o contexto cultural desempenha um papel importante na prática clínica e na eficácia do tratamento (Poremski et al.,2019). Esses autores apontam a prevalência de artigos em inglês nas principais bases de dados e a dificuldade para aprovação daqueles produzidos por outras culturas. No psicodrama, com múltiplas tendências e áreas de atuação, a diversidade cultural trazida pela participação de autores brasileiros é uma importante contribuição para o desenvolvimento da abordagem.

Para uma atualização em consonância com o que vem sendo desenvolvido no exterior, é fundamental que os brasileiros incluam publicações recentes, inclusive em inglês, em suas revisões do tema de estudo antes de iniciar a escrita. Essa etapa da escrita científica é considerada, no mundo da ciência, um requisito para a qualidade do artigo e da produção científica, gerando a possibilidade de ser citado nas principais revistas internacionais revisadas por pares.

A maioria dos interessados em publicar recebe uma formação limitada para a escrita científica. A expectativa é que aprenda a escrever artigos científicos "escrevendo", sem uma preparação formal (Fernández et al., 2018). Esses autores avaliaram a satisfação de alunos espanhóis de graduação e pós-graduação participantes de um programa intenso de dois dias de capacitação para a escrita científica e os resultados confirmaram a importância dessa disciplina.

Para os autores que não tem o inglês como idioma primário, o desafio para publicar em periódicos internacionais fica ainda maior. Um exemplo de abordagem dessa limitação foi a experiência da Universidade Médica de Tóquio, no Japão, que desenvolveu uma plataforma própria para submissão de manuscritos de seus alunos. Todos os manuscritos passam por um processo de consultoria e edição por professores altamente qualificados e experientes. Ao mesmo tempo, o aluno recebe material didático para a escrita científica (Barroga \&Mitoma,2019). Bons recursos tecnológicos facilitam a administração das contribuições, mas não substituem a dedicação de tempo por alunos e docentes na definição do foco do artigo, redação, revisão após as recomendações dos avaliadores e a finalização do manuscrito.

Um grupo de médicos e pesquisadores americanos de origem árabe organizou um curso on-line de redação e publicação científica para que colegas da Síria pudessem relatar os efeitos da guerra e suas condições adversas sob a perspectiva médica (Sabouni et al., 2017). Inicialmente, 2.588 médicos e estudantes de medicina aderiram ao grupo criado na mídia social, 385 completaram o questionário inicial e apenas 159 finalizaram o programa. Esses resultados sugerem que o tópico desperta interesse, mas que há impedimentos para adesão plena à tarefa.

Iniciativas como essas relatadas sugerem possibilidades de ação em favor da diversidade, equidade e inclusão (DEI) de autores relevantes no cenário internacional. Publicar bons artigos em inglês tem sido cada vez mais imperioso para o desenvolvimento de qualquer área de estudo, inclusive o psicodrama. Atingir o coletivo de pesquisadores e estudiosos de todos os continentes garante a atualidade do saber científico e mais oportunidades de troca entre diferentes tendências teóricas. 
O movimento atual para alavancar DEI na editoração científica (Fleury, 2021) deve contemplar programas de capacitação para a escrita e para a avaliação científica. O objetivo desse editorial é refletir sobre a importância da indexação e da internacionalização da Revista Brasileira de Psicodrama, assim como revisar algumas práticas para melhorar as habilidades de escrita científica.

\section{INDEXAÇÃO E INTERNACIONALIZAÇÃO}

A avaliação da qualidade de uma publicação científica baseia-se principalmente em critérios de indexação e citação. Indexação é o processo de inclusão de periódicos científicos em determinada base de dados, como, por exemplo, a Coleção SciELO. Como as mais importantes bases de dados definem critérios para esse processo, participar de uma delas tende a ser um atestado de qualidade reconhecido pela comunidade internacional.

Um desses critérios baseia-se no fator de impacto da revista, relativo à frequência com que um artigo é citado durante determinado período (Kieling \& Gonçalves, 2007). A visibilidade dos artigos é uma condição necessária para aumentar o número de citações e, consequentemente, influenciar positivamente seu fator de impacto.

As políticas editoriais, com várias regulamentações, e as novas propostas de ciência aberta no processo de avaliação têm estimulado o avanço na qualidade da pesquisa e da publicação científica (Gasparyan et al., 2018). Como os pesquisadores e autores utilizam mecanismos de busca de artigos em bases de dados reconhecidas para atualização, a indexação traz importantes implicações para a pesquisa e para o desenvolvimento de uma área de estudo (Gasparyan \& Kitas, 2021).

A Revista Brasileira de Psicodrama foi indexada na Coleção SciELO e a publicação passará a ser contínua, permitindo que os artigos possam chegar aos leitores com maior rapidez e ser mais citados. Como a indexação abre portas para o acesso a novas bases de dados internacionais, facilitando a apresentação dos artigos publicados a uma nova audiência, esse foi um passo decisivo para a internacionalização das diferentes tendências teóricas e práticas do psicodrama influenciado pela cultura brasileira.

\section{OS DESAFIOS DA ESCRITA CIENTÍFICA}

A análise de instruções aos autores de vários periódicos científicos, de 1987 a 2017, por meio de revisão sistemática e metanálise identificou o detalhamento crescente dos itens ao longo do tempo, principalmente em revistas com maior fator de impacto, da área da saúde e aquelas mais generalistas, diferenças entre países e entre bases de dados (Malički et al., 2021). Com esses critérios de análise, Malički et al. (2021) sugeriram consultoria profissional para atender às exigências crescentes, o que foi adotado pela Revista Brasileira de Psicodrama nos últimos anos.

Porém não bastam instruções detalhadas aos autores. Uma das principais limitações da Revista Brasileira de Psicodrama para novas e melhores indexações é a pouca familiaridade da comunidade psicodramática com a escrita científica, uma das possíveis explicações para o baixo número de citações dos artigos em outros periódicos. Como em outras revistas científicas, a capacitação dos autores e dos avaliadores para a escrita científica é essencial.

Em relação à atividade de avaliação, a Associação Brasileira de Editores Científicos (ABEC Brasil) oferece um curso de Ensino à Distância "Avaliador de Artigo Científico" com importantes subsídios para o aprimoramento das avaliações, inclusive com orientações para a implantação de um processo de avaliação em ciência aberta. O diferencial desse curso é o aprendizado com exercícios práticos simulando a avaliação por pares.

Em relação aos autores, a maioria deles tem dificuldade em atender as especificações para cada seção do artigo, a função dos componentes do resumo (introdução, objetivos, método, análise e discussão e considerações finais) e em estabelecer critérios de inclusão e exclusão em artigos de pesquisa de campo e de revisão. Considerando a imperiosidade de alavancar os princípios DEI na Revista Brasileira de Psicodrama, novas referências precisam ser buscadas para atender às limitações de nossos autores com a escrita científica.

As recomendações gerais para a escrita científica sugerem iniciar com a leitura dos principais artigos publicados anteriormente sobre o assunto, com uma revisão bibliográfica adequada. Mesmo reconhecendo a dificuldade intrínseca dessa tarefa, Forero et al. (2020) ressaltam que a ciência avança indo além do que é conhecido. Essa tarefa exige tempo e disciplina, um obstáculo frequente entre autores potenciais. 
A International Association for Group Psychotherapy and Group Processes (IAGP) promoveu um grupo de preparação para a escrita científica com participantes de vários países. A partir de uma série de dicas (Grundy, 2021), o compartilhamento dos textos e das dificuldades para a escrita científica vem estimulando novas publicações.

O artigo científico deve ser curto, compacto e simples, evitando o uso excessivo de adjetivos e advérbios (Forero et al., 2020). Os tópicos básicos para melhorar a escrita científica de autores de outros idiomas são, entre outros, o uso correto da gramática, estilo editorial/científico, estrutura de sentenças, componentes do artigo científico, comunicação em congressos, preparação do artigo para publicação (Barroga \& Vardaman, 2015).

\section{CONCLUSÃO}

Com a inclusão da Revista Brasileira de Psicodrama na Coleção SciELO, o comitê editorial está em busca de novos parâmetros de cientificidade para alcançar patamares mais altos de qualidade, tanto na política editorial como na capacitação dos interessados em publicar e em avaliar os manuscritos submetidos à revista, com o intuito de aprender sempre e desenvolver um processo de aprendizagem reflexiva e atualizada.

Desenvolvimentos teóricos e práticos do psicodrama, influenciados pela rica cultura brasileira, tem muito a contribuir, principalmente em países em desenvolvimento. A indexação na Coleção SciELO iniciou esse processo, com o acesso aberto aos artigos, alguns deles em inglês.

Nessa próxima década, a capacitação para a escrita científica e para a publicação em inglês torna-se imperiosa para o compartilhamento da diversidade cultural, da equidade entre psicodramatistas e a inclusão cada vez maior de autores de diferentes tendências teóricas e de áreas afins que possam contribuir para novos desenvolvimentos, condições essenciais para a internacionalização do psicodrama brasileiro.

\section{REFERÊNCIAS}

Barroga, E. \& Mitoma, H. (2019). Improving Scientific Writing Skills and Publishing Capacity by Developing University-Based Editing System and Writing Programs. Journal of Korean Medical Science, 34(1), e9. https://doi.org/10.3346/jkms.2019.34.e9

Barroga, E. \& Vardaman, M. (2015). Essential Components of Educational Programs on Biomedical Writing, Editing, and Publishing. Journal of Korean Medical Science, 30(10), 1381-1387. https://doi.org/10.3346/jkms.2015.30.10.1381

Fernández, E., García, A. M., Serés, E. \& Bosch, F. (2018). Students' satisfaction and perceived impact on knowledge, attitudes and skills after a 2-day course in scientific writing: a prospective longitudinal study in Spain. BMJ Open, 8(1), e018657. https:// doi.org/10.1136/bmjopen-2017-018657

Fleury, H. J. (2021). O psicodrama confirma missão política da diversidade, equidade e inclusão. Revista Brasileira de Psicodrama, 29(3), 159-162. https://doi.org/10.15329/2318-0498.00509_PT

Forero, D. A., Lopez-Leon, S. \& Perry, G. (2020). A brief guide to the science and art of writing manuscripts in biomedicine. Journal of Translational Medicine, 18, 425. https://doi.org/10.1186/s12967-020-02596-2

Gasparyan, A. Y., Yessirkepov, M., Voronov, A. A., Koroleva, A. M. \& Kitas, G. D. (2018). Updated Editorial Guidance for Quality and Reliability of Research Output. Journal of Korean Medical Science, 33(35), e247. https://doi.org/10.3346/jkms.2018.33.e247

Gasparyan, A. Y. \& Kitas, G. D. (2021). Editorial Strategy to Get a Scholarly Journal Indexed by Scopus. Mediterranean Journal of Rheumatology, 32(1), 1-2. https://doi.org/10.31138/mjr.32.1.1

Grundy, D. (2021). Tips on professional writing [Manuscrito não publicado]. Professional Writing Course, International Association for Group Psychotherapy and Group Processes (IAGP).

Kieling, C. \& Gonçalves, R. R. F. (2007). Assessing the quality of a scientific journal: the case of Revista Brasileira de Psiquiatria. Brazilian Journal of Psychiatry, 29(2), 177-181. https://doi.org/10.1590/S1516-44462007000200017

Malički, M., Jerončić, A., Aalbersberg, I. J., Bouter, L. \& Riet, G. (2021). Systematic review and meta-analyses of studies analysing instructions to authors from 1987 to 2017. Nature Communications, 12, 5840. https://doi.org/10.1038/s41467-021-26027-y

Poremski, D., Falissard, B., Fegert, J., Witt, A., Ordóñez, A. E., Martin, A., Fung, D. S. S. (2019). Moving from 'personal communication' to 'available online at': preprint servers enhance the timeliness of scientific exchange. Child and Adolescent Psychiatry and Mental Health, 13, 42. https://doi.org/10.1186/s13034-019-0301-4

Sabouni, A., Chaar, A., Bdaiwi, Y., Masrani, A., Abolaban, H., Alahdab, F., ... Al-Moujahed, A. (2017). An online academic writing and publishing skills course: Help Syrians find their voice. Avicenna Journal of Medicine, 7(3), 103-109. https://doi.org/10.4103/ ajm.AJM_204_16 